\title{
Use of online resources in the taxonomy and ecology of harmful phytoplankton
}

\author{
Alexandra C. Kraberg ${ }^{1 \dagger *}$ and David J. S. Montagnes ${ }^{2}$ \\ ${ }^{1}$ University of Liverpool, School of Biological Sciences, Port Erin Marine Laboratory, Isle of Man, IM9 6JA, British Isles, \\ and 2 University of Liverpool, School of Biological Sciences, BioSciences Building, Crown Street, Liverpool, L69 7ZB, UK
}

\section{SUMMARY}

The internet is becoming increasingly important for the dissemination of biological information. Large target audiences are reached rapidly, large amounts of information can be assembled and distributed, sites can include search facilities, and information might be handled online or as printable output. Many websites are now producing libraries of phytoplankton images including toxic dinoflagellates. But while some provide valuable resources others lack focus and do not address any specific problems, for example the morphological variability in many major groups such as Dinophysis and Alexandrium. Yet it is this morphological variation which often hinders taxonomic and ecologic research of harmful dinoflagellates. Taxonomic information as well as information about sample origin and preservation methods is also often lacking. This makes comparisons between taxonomic websites difficult. Therefore, while documenting the presence of certain taxa, some sites cannot be used as a taxonomic training or research tool. Yet, if online resources were to reflect these complexities they could become valuable tools in the dissemination of data as well as actual research and training tools. One reason for the lack of consistency between sites could be that these sites, as a teaching and research tool, are a relatively recent development and no criteria have therefore been established as to the contents and structure of such sites, as are commonplace for conventional publications. This comment will discuss different approaches to, and tools for, the production of online taxonomic resources (depending on the site's objectives, e.g. research or teaching) using the Harmful Plankton Project (http:// www.liv.ac.uk/hab) designed at the University of Liverpool as one example of a user-friendly and adaptable tool.

Key words: ecology, harmful dinoflagellates, internet, online guides, phytoplankton, taxonomy.

\section{INTRODUCTION}

The internet is becoming increasingly important in scientific debate and the dissemination of data. The use of the internet for data dissemination has distinct advantages over conventional methods in that information can be displayed several different ways, can be made available more quickly and be communicated to a greater number of users (with a wider range of professional backgrounds) than with the conventional publication process. Online resources can also include efficient search facilities for information within a specific site or between different online resources. Moreover, information can be organized for both online study and as print versions. The internet is, therefore, an ideal medium for the dissemination and handling of data and the development of teaching tools in rapidly evolving fields of study such as dinoflagellate taxonomy and ecology.

Dinoflagellate taxonomy, in particular, has undergone radical changes in the past few decades with new methods leading to a large number of changes in species/genus names and the creation of new genera. In fact, the morphological species concept itself has been challenged on the basis of recent genetic evidence (Costas et al. 1995; Osborn et al. 2000). These advances will also have implications for future assessments of dinoflagellate biodiversity and ecology and for the production of taxonomic tools for dinoflagellate identification. It is essential that these changes, but also ambiguities and conflicts in the designation of species names, are communicated in a structured manner. Not all such changes are accepted readily by the wider scientific community. Here again, the internet can be an ideal medium, as online taxonomic resources can be designed to include discussion forums and mailing lists to ensure all criticisms and comments about taxonomic problems can be voiced and discussed.

The problems with respect to dinoflagellate taxonomy are further confounded by methodological problems such as the different appearance or degree of preservation of cells in different fixatives.

*To whom correspondence should be addressed.

tPresent address: Biologische Anstalt Helgoland (Alfred-

Wegener Institut für Polar- und Meeresforschung), 201

Kurpromenade, 27498 Helgoland.

Email: akraberg@awi-bremerhaven.de

Communicating editor: T. Horiguchi

Received 30 December 2003; accepted 13 July 2004. 
The constantly evolving concepts surrounding dinoflagellate biology and ecology, together with the substantial degree of variation in dinoflagellate morphology on different spatial and temporal scales as well as possible identification problems arising from different preservation methods, provide a perfect reason for making use of the strengths of the internet to create flexible, user-friendly online resources that can become true taxonomic/ecologic teaching and analysis tools and can complement offline standard resources such as those by Dodge (1982) and Hallegraeff et al. (2003). Such tools will not only provide a data dissemination device but also an identification aid and can generally facilitate faster progress in dinoflagellate research by providing more efficient links and access to different types of taxonomic and ecologic information and by facilitating collaborative projects. Some good, illustrated guides to phytoplankton and protists already exist (e.g. http://www.mbl.edu/microscope, http://www.marbot.gu.se, http://dinos.anesc.u-tokyo.ac.jp); although, with the exception of the latter, they only have limited mechanisms, such as identification keys, for actually identifying taxa.

However, the internet's flexibility, meaning here the opportunity to view many resources simultaneously and to arrange and select information according to the user's requirements, can also make using the internet a frustrating experience, because of the multitude of information available and the large number of linkages within and between sites. This can make organizing the available information and making comparisons between different websites difficult or even impossible.

Therefore, a true online taxonomic tool needs a clear concept resulting in an efficient navigation structure within the site and the potential for useful sharing of information and comparison between different sites. This requires the definition of a set of criteria for the biological and technical requirements of such sites to enable their use as teaching and research tools. Use of some general criteria in future sites could facilitate better comparability between sites and would leave the potential to extend these sites to an online database format (e.g. in the form of distributed databases) in the future. Online taxonomic resources are a relatively recent development and such criteria have not yet been established. But as a general rule, they should still allow individual sites to develop their own identity (e.g. providing a local geographic perspective), just as different journals are clearly distinguishable but also apply common standards that allow comparisons between them.

This comment deals with one example of an internet resource, the Harmful Plankton Project at the University of Liverpool, UK which attempts to provide a structured online resource that uses a set of concrete criteria that might be used in future to produce similar tools. The suggested site structure and type of information can

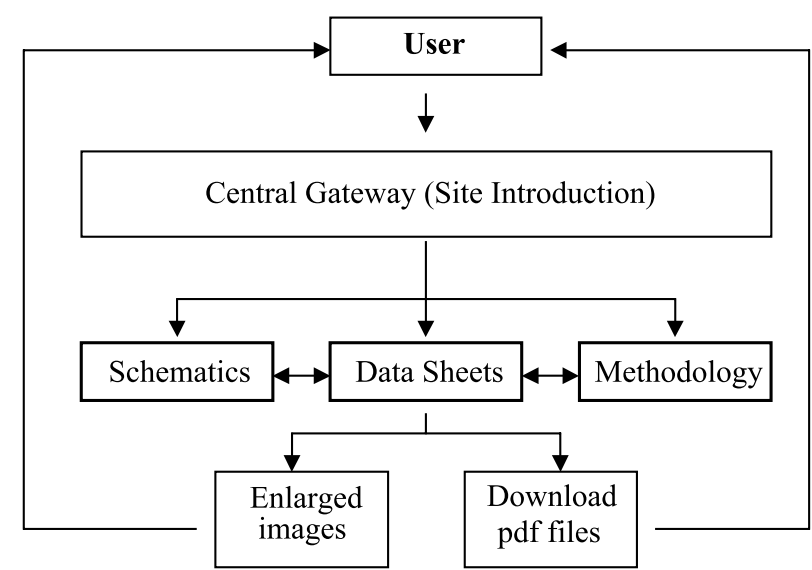

Fig. 1. Schematic representation of the general concept of the guide to harmful phytoplankton including the navigation pathways between the different modules of the website.

facilitate better comparison between sites and make the site versatile enough to be adapted easily to other species groups and to be incorporated into online databases. The site provides standardized text sheets with information of image origin and methodologies used, and also aims to supply, wherever possible, images of live and differently preserved cells for each species. It provides the opportunity to arrange and view different types of information simultaneously, as chosen by the user, so that different types of users should find the site accessible and useful. Importantly, it also contains a facility for users to communicate criticisms and comments to the site owners.

\section{The general concept of the Harmful Plankton Project}

The Harmful Plankton Project website was designed with several major goals in mind. In terms of the science it aims at being comprehensive. This includes providing several drawings and images (using different preservation methods) per species as well as detailed descriptions of both taxonomy and ecology. Two further criteria were user-friendliness and flexibility. This flexibility, in terms of choosing the information to be displayed, is particularly important as access to taxonomic and ecologic information is sought not only by scientists in the respective field, but also environmental managers and other professionals involved, in large scale monitoring studies for example. To accommodate these needs, a modular approach was chosen as the general site concept (Fig. 1). This not only means flexibility in arranging information but also keeps individual pages short, therefore avoiding the need for excessive scrolling, which might discourage the site user from viewing all the information presented on a particular page (Wang et al. 2000). Using this modular approach, a clear navigation structure could be created 

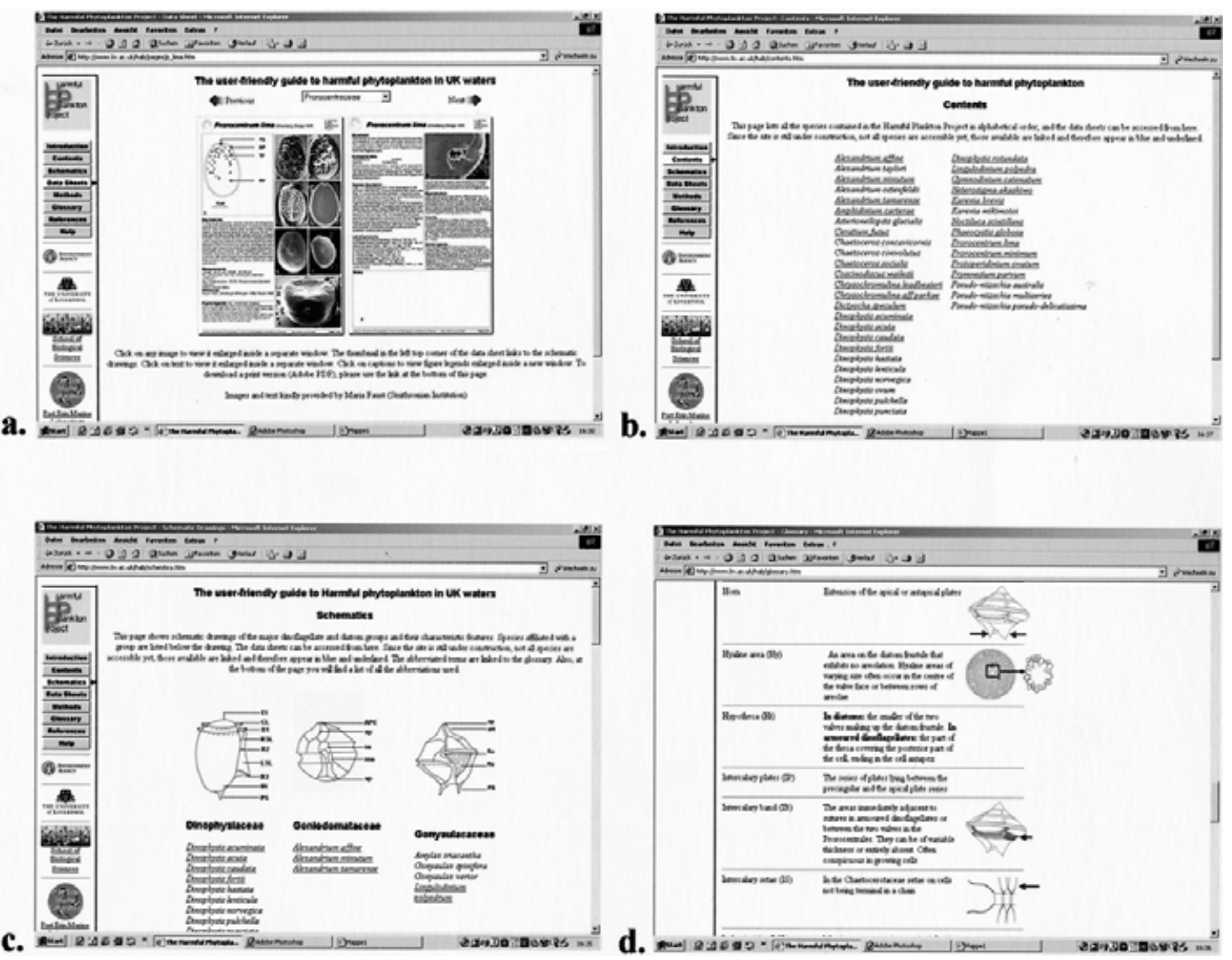

Fig. 2. (a)-(d) The main pages in the Harmful Plankton Project: (a) species page, showing all the information available for this species in a condensed form, and (b) contents page, allowing access to individual data sheets by clicking on the species name, (c) schematics section, (d) glossary. Each page allows access to all other pages by the left-hand gray navigation buttons, and clicking on abbreviations in the schematics section will also link to the glossary.

to access and retrieve the detailed taxonomic and ecologic information from different parts of the site. This modular structure works within the site by creating separate modules for methodology, data sheets, schematics, glossary and references (all of which are interlinked; Fig. 1). It also works externally by creating a template that can be adapted to different species groups, producing different 'site modules' that can be linked through an online database.

The nature of the information displayed means that several parts of the site might need to be displayed simultaneously. Therefore, the site has been designed: (i) to show the main species pages in one window (Fig. 2a), with enlargements of each image, text and caption viewable in a separate window, which could be particularly important for comparing dinoflagellates with their large number of morphologically similar species; and (ii) to provide constant access to any additional information such as glossaries, schematics and help screens through the gray navigation bar visible throughout the site. The detailed species data sheets (containing schematics and images) can be accessed in three ways, depending on the previous knowledge of the user: by clicking on the species name on the contents page (Fig. 2b), by browsing through the schematics page displaying characteristic drawings for the species groups represented in the site (Fig. 2c) or by using the drop-down menu and orange browse buttons on each of the data sheets. The species descriptions on the data sheets are further complemented with ecological information to place each species in the context of its natural environment. Finally, all information can be printed or downloaded as pdf files. Along with traditional taxonomic texts, the site also provides glossaries with definitions of terminology and simplified drawings explaining the basics of dinoflagellate and diatom taxonomy (Fig. 2d). Having recognized the importance of the internet for enhancing communication, the site also provides the opportunity for user comments to the site owners and has produced a mailing list.

This set-up allows different types of users to select the information most pertinent to their needs and arrange it side by side on the computer screen, facilitating a review of the information for a single species or indeed comparisons between morphologically similar 


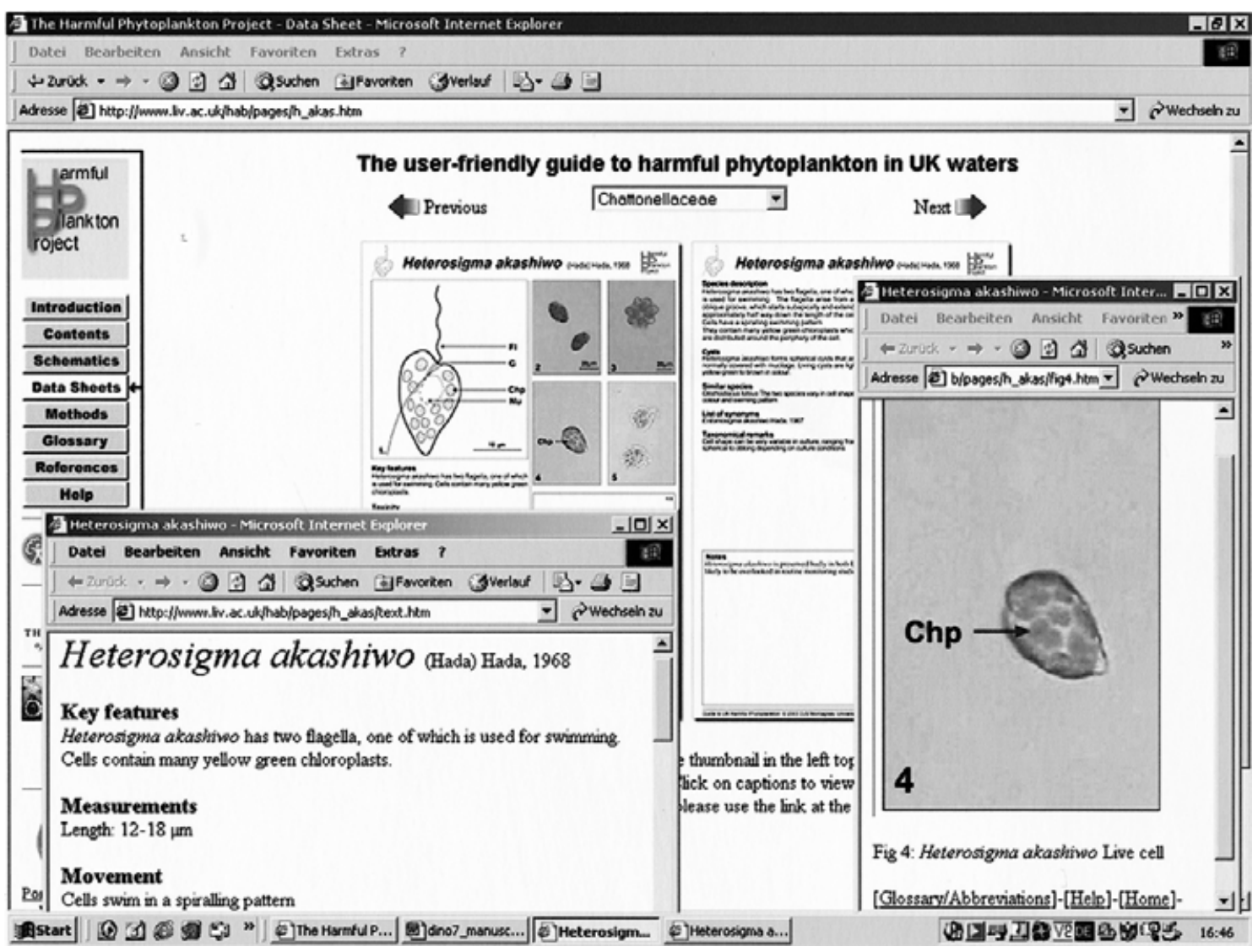

Fig. 3. Example of a screen with species description and enlarged version of image opened in separate window and arranged side by side to satisfy specific user needs.

species (Fig. 3). In addition, the chosen site structure has made site navigation very adaptable, so that it can easily be modified for different species groups, for example by changing the categories used in the species descriptions.

\section{DISCUSSION AND CONCLUSION}

One reason commonly cited for not using online taxonomic resources as true identification aids alongside traditional journals and books is that pages on the internet are not peer reviewed in the same way as scientific papers; although, potentially, each page might be critically viewed by thousands of visitors a day. Since internet resources have rarely been used as true taxonomic tools, criteria that determine the requirements of such sites to facilitate their acceptance and routine use by different user groups have not yet been established. Different users will have different requirements, and the site structure has to be adaptable enough to satisfy these needs. A debate of what these requirements are, is still in its infancy but preliminary discussions and results of a survey recently carried out among colleagues indicate that there is a clear separation into biological and technical factors, with technical factors being regarded as less important than biological ones such as multiple images per species using different preservation methods.

Since one of the main components of the conventional method of the manuscript publication process; namely, the peer review, is lacking in online resources, it might be expected that the presence of an editor in overall charge of such resources would be ranked very highly; however, early discussions with a range of scientists indicate that this might not hold true. It might be more important to address specific scientific problems with an appropriate concept so that the success of the site can be assessed and criticisms and comments be voiced succinctly, providing a novel, public review process. The success of the site and the intensity of critical reviews will then go hand in hand. This kind of debate should go on in the wider scientific community and it is hoped that this short comment and the website we have described (http://www.liv.ac.uk/ hab) can help to develop this debate.

The production of such sites is not suggested to replace the conventional process of data dissemination. However, if designed appropriately, they can complement and enhance traditional resources to the benefit of researchers and environmental managers alike.

The present site is only one of many ways of implementing such resources. However, the site structure used 
for the Harmful Plankton Project site has shown itself to be adaptable enough to be used for two very different groups of organisms. It is already the second site using this modular structure, with the first site using the same layout being the guide to coastal ciliates (Strueder-Kypke et al. 2000-2001; StruederKypke \& Montagnes 2002). This shows the adaptability of this resource (Strueder-Kypke et al. 2000-2001; Strueder-Kypke \& Montagnes 2002) and the usefulness of the concept as a whole. Interest has already been expressed to extend the site concept to groups such as copepods. Our next aim will be to underpin the site with a true database structure from which further information can be retrieved at the control of the site user, making it even more flexible and user-friendly. The planned database will then also form a connection between the sites that are based on our template, starting with the Harmful Plankton Project and the Coastal Planktonic Ciliate Project websites.

We feel it is essential that the production of ground rules for the creation of such large-scale tools be a collaborative effort, so that resources benefit the maximum number of users and ensure the long-term existence of these sites in the future. Incorporating online elements into future research projects can support the research but might also open up new funding opportunities and, in the long term, reduce the costs of data dissemination.

The production of the Harmful Plankton project website has already been a collaborative effort with advice and material provided by several scientists. Moreover, its modular nature provides an ideal opportunity for further collaborations by adding modules for different species groups and linking these through an online database structure. We invite collaborations in such projects and comments and criticisms regarding the site concept.

\section{ACKNOWLEDGMENTS}

The authors wish to thank all colleagues who provided images and/or text for the Harmful Plankton Project, particularly Maria Faust (Smithsonian Institution) for provision of images and descriptions and Gianfranco Novarino (Natural History Museum, London) for provision of images, whose help was greatly appreciated. Partial funding for creation of the site was provided by the UK Environment Agency. Thanks are also due to the organizers of the DINO 7 conference.

\section{REFERENCES}

Costas, E., Zardoya, R., Bautista, J., Garrido, A., Rojo, C. and Lopez-Ródas, V. 1995. Morphospecies vs. genospecies in toxic marine dinoflagellates: an analysis of Gymnodinium catenatum/ Gyrodinium impudicum and Alexandrium minutum/ A. Iusitanicum using antibodies, lectins, and gene sequences. J. Phycol. 31: 801-7.

Dodge, J. D. 1982. Marine Dinoflagellates of the British Isles. Her Majesty's. Stationary Office, London, 142 pp.

Hallegraeff, G. M., Anderson, D. A. and Cembella, A. 2003. Manual on Harmful Microalgae. UNESCO Publishing, Paris.

Osborn, A. M., Moore, E. R. B. and Timmis, K. N. 2000. An evaluation of terminal-restriction fragment length polymorphism (T-RFLP) analysis for the study of microbial community structure and dynamics. Environ. Microbiol. 2: 39-50.

Strueder-Kypke, M. C., Kypke, E. R., Agatha, S., Warwick, J. and Montagnes, D. J. S. 2000-01. The user-friendly guide to coastal planktonic ciliates (online). University of Liverpool, Liverpool. Available from URL: http://www.liv.ac.uk/ ciliate (date of access 01-02-2002).

Strueder-Kypke, M. C. and Montagnes, D. J. S. 2002. Development of web-based guides to planktonic protists. Aquat. Microb. Ecol. 27: 203-7.

Wang, P., Hawk, W. B. and Tenopir, C. 2000. Users' interaction with worldwide web resources: an exploratory study using a holistic approach. Inf. Process. Manage. 36: 229-51. 